\title{
riccafd
}

Revista Iberoamericana de Ciencias de la Actividad Física y el Deporte

\section{EL EJERCICIO FÍSICO EN EL AULA PARA LA MEJORA DEL COMPORTAMIENTO DE NIÑOS AUTISTAS}

\section{PHYSICAL EXERCISE IN THE CLASSROOM TO IMPROVE THE BEHAVIOR IN AUTISTIC CHILDREN}

Suárez-Manzano, Sara ${ }^{1}$; López-Serrano, Sebastián ${ }^{1}$; Belchior de Oliveira, Polyanna ${ }^{1}$; da Cruz Murta, Luis Manuel ${ }^{2}$

Suárez-Manzano, Sara. Departamento de Didáctica de la Expresión Musical, Plástica y Corporal. Facultad de Humanidades y Ciencias de la Educación. Universidad de Jaén (España). López-Serrano, Sebastián. Departamento de Didáctica de la Expresión Musical, Plástica y Corporal. Facultad de Humanidades y Ciencias de la Educación. Universidad de Jaén (España). Belchior de Oliveira, Polyanna. Departamento de Didáctica de la Expresión Musical, Plástica y Corporal. Facultad de Humanidades y Ciencias de la Educación. Universidad de Jaén (España). da Cruz Murta, Luis Manuel. Department of Arts, Humanities and Sports. Polytechnic Institute of Beja (Portugal).

Dirección para correspondencia: Sara Suárez Manzano. ssuarez@ujaen.es DOI: http://dx.doi.org/10.24310/riccafd.2019.v8i1.5792

\section{RESUMEN}

El objetivo de este trabajo fue analizar todos los estudios que midieron el efecto a medio plazo de intervenciones educativas basadas en ejercicio físico sobre el comportamiento y conducta de niños (6 - 12 años de edad) diagnosticados con Trastorno del Espectro Autista (TEA). Para ello se hizo una revisión bibliográfica en tres bases de datos (búsqueda inicial $n=184$ : PubMed $n=24$, SportDiscus $\mathrm{n}=98$, Web of Science $\mathrm{n}=62$ ). Se limitaron las fechas de búsqueda desde enero de 2000 hasta enero de 2018. Un total de cinco estudios longitudinales con inter-vención cumplieron los criterios de inclusión. Todos los estudios son recientes, el más antiguo fue publicado en 2011. Los cinco trabajos emplearon programas de ejercicio físico en el ámbito escolar - 3-5 días / semana - con duración de 2-20 semanas. Las sesiones realizadas en días de clase tuvieron una duración de 15-30 minutos, las realizadas en vacaciones escolares cuatro horas. Tres estudios obtuvieron mejoras en comportamiento tras la intervención educativa, sin embargo, dos estudios no obtuvieron cambios significativos en las variables medidas. Se concluye que la integración del ejercicio físico en el ámbito escolar mejora el comportamiento y conducta (intereses restrictivos o repetitivos y com-portamientos estereotipados) en niños diagnosticados TEA. Como perspectivas futuras se sugiere profundizar en la influencia de los factores de confusión y en el efecto diferencial del efecto a 
corto, medio y largo plazo de estas intervenciones educativas basadas en el ejercicio físico.

Palabras clave: actividad física, condición física, comportamiento, colegio, revisión.

\section{ABSTRACT}

The aim of this work was to analyze all the studies that measured the medium term effect of educational interventions based on physical exercise on the behavior of children (6 - 12 years of age) diagnosed with Autism Spectrum Disorder (ASD). A bibliographic review was done in three databases (initial search $n=184$ : PubMed $n=24$, SportDiscus $n=98$, Web of Science $n=62$ ). Search dates were limited from January 2000 to January 2018. A total of five longitudinal studies with intervention met the inclusion criteria. All the studies are recent, the oldest was published in 2011. The five studies used physical exercise programs in the school setting - 3-5 days / week - with duration of 2-20 weeks. The sessions carried in class days lasted 15-30 minutes, the ones realized in school holidays, four hours. Three obtained improvements in behavior after the educational intervention, however two studies did not obtain significant changes in the measured variables. It is concluded that the integration of physical exercise in the school environment improves behavior (restrictive or repetitive interests and stereotyped behaviors) in children with ASD. As future perspectives we suggest to deepen the influence of the confounding factors and the differential effect of the short, medium and long term effect of these educational interventions based on physical exercise.

Key words: Physical activity, physical condition, behavior, school, review.

\section{INTRODUCCIÓN}

El Trastorno del Espectro Autista (TEA) se caracteriza por anomalías en las interacciones sociales, deficiencias en el lenguaje y la comunicación, intereses restrictivos o repetitivos y comportamientos estereotipados (DSM5, 2013). Según el DSM5 (2013), el diagnóstivo TEA se subdivide en tres subtipos: el trastorno autista, el síndrome de Asperger y el trastorno generalizado del desarrollo no especificado. La tasa de prevalencia mundial del TEA en niños de entre seis y 12 años es aproximadamente del 0,70\% (Lai, Lombardo y Baron-Cohen, 2014).

Para modificar el comportamiento, el tratamiento psicológico más habitual es la modificación de conducta, dirigida a enseñar a los jóvenes a ser conscientes y controlar su comportamiento, trabajando el autocontrol (Heyvaert, Saenen, Campbell, Maes y Onghena, 2014). Como complemento, en los últimos años se sugiere la práctica de actividad física (AF). Anderson-Hanley, Tureck y Schneiderman (2011), observaron en 24 adolescentes autistas (10-18 años) mejoras significativas en comportamientos repetitivos y función ejecutiva tras jugar 20 minutos con videojuegos activos. Estos cambios podrían deberse al efecto de 
la práctica de AF y ejercicios de relajación, al disminuir los niveles de cortisol atenuando como consiguiente el estado de ansiedad y estrés (Hillier, Murphy y Ferrara, 2011).

A pesar de lo anterior, los efectos de la práctica de AF sobre el comportamiento en niños autistas no son concluyentes. Las revisiones conocidas no especifican el momento de realización de la intervención o no limitan las poblaciones a solo niños (6-12 años) y en ningún caso se centraron los trabajos de revisión en intervenciones asociadas a la jornada escolar de los niños (Sowa y Meulenbroek, 2012; Tan, Pooley y Speelman, 2016). Por lo tanto, la pregunta para este estudio fue la siguiente: "¿La práctica de ejercicio físico asociadas a la jornada escolar mejora la conducta y comportamiento de los niños Autistas?". Este trabajo está focalizado en niños TEA, porque la niñez es la etapa vital idónea para crear adherencia a la práctica de actividades físico-deportivas (Ortega et al., 2011). Pensamos que los resultados pueden ser útiles para conocer las aportaciones educativas que el ejercicio puede ofrecer a los niños autistas, pudiendo así guiar a familiares, docentes y monitores para aprovechar sus beneficios, tanto en el ámbito extraescolar, como en el educativo.

\section{MÉTODO}

El método seguido para este estudio fue la revisión sistemática, basándonos en los estándares propuestos por Sánchez-Meca (2010). En primer lugar, se determinaron las palabras de búsqueda, límites de búsqueda y criterios de inclusión. Dos investigadores realizaron la búsqueda por separado. A continuación, se compararon los resultados obtenidos, descartando aquellos artículos duplicados y los que no cumplían los límites de búsqueda basándonos en título y resumen. Finalmente, siguiendo los criterios de inclusión, se seleccionaron los estudios que finalmente se incluirían en el trabajo.

\section{Límites de búsqueda}

La búsqueda se realizó en tres bases de datos (PubMed, SportDiscus y Web of Science). Limitando la fecha de publicación desde enero del 2000 hasta marzo del 2017, debían de ser artículos escritos en inglés, publicados en revistas indexadas con revisión por pares. Los términos de búsqueda fueron:

1) Physical activity, physical education, exercise, physical exercise.

2) Autism spectrum disorder, autism spectrum, autism, Asperger, ASD.

3) Children, school-age, student, school.

\section{Criterios de inclusión}

Los criterios que debían cumplir los artículos para ser incluidos en el presente trabajo de revisión fueron:

$1^{\circ}$ Informe completo publicado en una revista revisada por pares.

$2^{\circ}$ La población del estudio fueron niños diagnosticados TEA (6-12 años). 
$3^{\circ}$ Trabajos a texto completo y escritos en inglés.

$4^{\circ}$ El estudio utilizó un diseño con intervención realizada por especialistas de la AF y Deporte.

$5^{\circ}$ No existen criterios de exclusión con respecto al origen étnico.

\section{Evidencia del nivel de calidad}

Todos los estudios incluidos fueron considerados de alta calidad, en base a otras listas de evaluación (Suarez-Manzano, Ruiz-Ariza, De La Torre-Cruz y Martínez-López, 2018). Para considerarse artículos de alta calidad, los artículos debían obtener una puntuación total entre 9-12. Las puntuaciones de cada apartado variaron entre cero-dos, calificando con cero puntos en el caso de no cumplir la condición, un punto si se cumplía parcialmente y dos puntos si se cumplía totalmente.

Tabla 1. Lista de estudios incluidos con puntuaciones de calidad.

\begin{tabular}{lllllllll}
\hline \multicolumn{1}{c}{ Autores } & A & B & C & D & E & F & Puntuación Total & $\begin{array}{c}\text { Nivel de } \\
\text { calidad }\end{array}$ \\
\hline Nicholson et al. (2011) & 2 & 2 & 2 & 1 & 2 & 0 & 9 & AC \\
\hline Oriel et al. (2011) & 2 & 2 & 2 & 1 & 2 & 0 & 9 & AC \\
\hline Vander y Sprong (2011) & 2 & 2 & 2 & 1 & 2 & 0 & 9 & AC \\
\hline Liu y Hamilton (2013) & 2 & 2 & 2 & 2 & 2 & 2 & 12 & AC \\
\hline Ketcheson et al. (2017) & 2 & 2 & 2 & 1 & 2 & 2 & 11 & . \\
\hline
\end{tabular}

Nota: Alta Calidad (AC)= 9-12.
A. Estudio publicado al completo en una revista revisada por pares y escrito en inglés.
B. La población estudiada fue niños (6-12 años) diagnosticados TEA.
C. Se describen claramente los resultados referentes a la variable comportamiento.
D. Se describe con claridad la intervención físico-deportiva.
E. La intervención se realiza en el ámbito escolar y por especialistas.
F. El estudio tenía un diseño intervencionista.

\section{RESULTADOS}

\section{Hallazgos generales}

El gráfico de flujo muestra las fases de selección de los artículos incluidos en este trabajo de revisión (Figura 1). En la búsqueda principal se obtuvieron 184 artículos. Se eliminaron 36 duplicados, quedando 148. En el siguiente paso se desestimaron 83, en base a los límites de búsqueda. Así, 65 estudios fueron revisados con los criterios de inclusión. En este último cribado se excluyeron un total de 60 artículos. Quedando finalmente cinco artículos aptos para ser incluidos en el presente trabajo de revisión. 


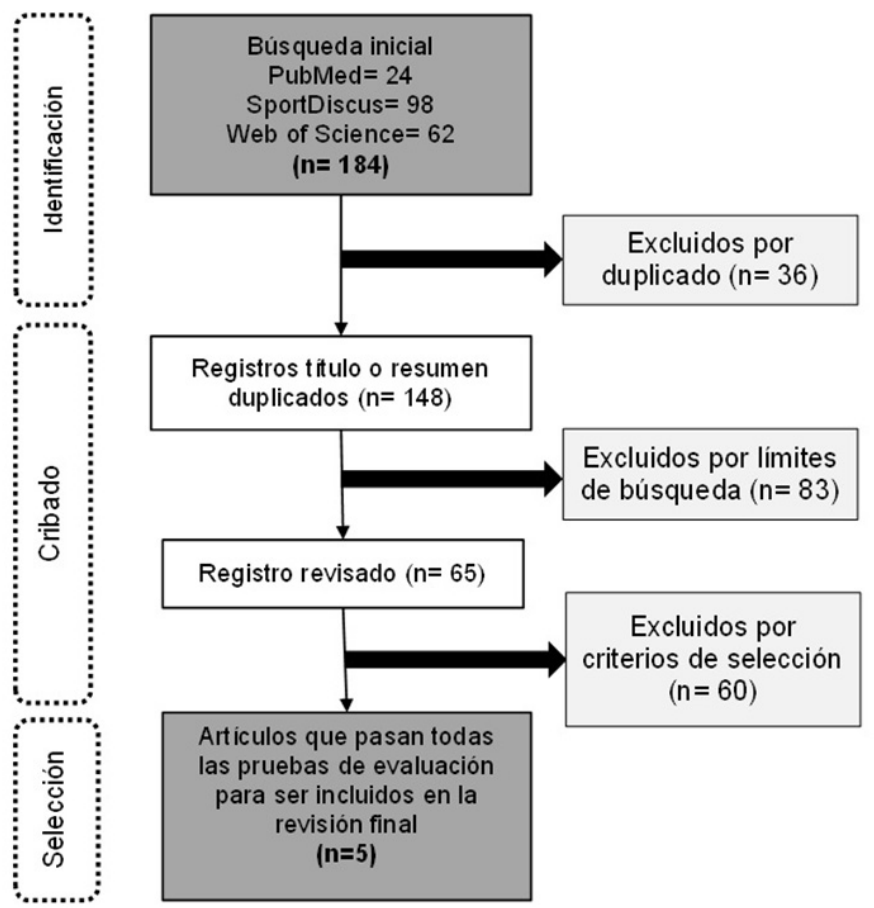

Figura 1. Gráfico de flujo del proceso de selección de artículos.

En este trabajo de revisión se han incluido cinco artículos, todos ellos con diseño de intervención (100\%), asignando a los participantes de forma aleatoria en los diferentes grupos. Todos ellos evalúan el efecto a medio plazo de un programa de AF, en el comportamiento de los niños autistas. Esta revisión incluye datos de 66 participantes, siendo el tamaño muestral de los diferentes estudios de entre cuatro (Nicholson et al., 2011) y 23 (Liu y Hamilton, 2016). Los cinco estudios se realizaron en USA.

Tabla 2. Cinco trabajos de los últimos siete años que evalúan el efecto de la AF en el comportamiento en niños autistas.

\begin{tabular}{|c|c|c|c|c|c|}
\hline Autor y año & $\begin{array}{l}\text { Diseño de } \\
\text { estudio/ } \\
\text { intervención }\end{array}$ & $\begin{array}{l}\text { Muestra/ } \\
\text { Edad/ } \\
\text { País }\end{array}$ & Grupos & $\begin{array}{l}\text { Medidas de } \\
\text { comportamiento }\end{array}$ & Resultados \\
\hline $\begin{array}{l}\text { Nicholson } \\
\text { et al. } \\
(2011)\end{array}$ & $\begin{array}{l}\text { Intervención/ } \\
\text { Diagnóstico de } \\
\text { TEA / } \\
2 \text { semanas. } \\
3 \text { días/ sem. } \\
\text { Antes de clase. }\end{array}$ & $\begin{array}{l}4 \text { niños/ } \\
\text { 9/ } \\
\text { USA }\end{array}$ & $\begin{array}{l}1 \text { grupo: } \\
\text { GE: TEA. } 12 \\
\text { min carrera } \\
\text { aeróbica + } 5 \\
\text { min vuelta a la } \\
\text { calma }\end{array}$ & $\begin{array}{l}\text { BOSS: } \\
\text { comportamiento } \\
\text { durante la tarea } \\
\text { académica }\end{array}$ & $\begin{array}{l}\text { Gran efecto en el } \\
\text { tiempo activo de } \\
\text { aprendizaje. Tras } \\
\text { la intervención } \\
\text { se muestran más } \\
\text { activos. }\end{array}$ \\
\hline
\end{tabular}




\begin{tabular}{|c|c|c|c|c|c|}
\hline $\begin{array}{l}\text { Oriel et al. } \\
\text { (2011) }\end{array}$ & $\begin{array}{l}\text { Intervención/ } \\
\text { Diagnóstico de } \\
\text { TEA/ } \\
3 \text { semanas + } \\
3 \text { semanas. } 5 \\
\text { días/sem. } \\
\text { Antes de clase }\end{array}$ & $\begin{array}{l}9 \text { chicos ( } 2 \\
\text { chicas)/ } \\
3-6 / \\
\text { USA }\end{array}$ & $\begin{array}{l}2 \text { grupos: } \\
\text { GE }(n=9) \text { : } \\
\text { 15min correr, } \\
\text { seguido de } \\
\text { tarea en el } \\
\text { aula. } \\
\text { GC }(n=9)\end{array}$ & $\begin{array}{l}\text { Comportamientos } \\
\text { estereotípicos y } \\
\text { comportamiento en } \\
\text { la tarea }\end{array}$ & $\begin{array}{l}\text { Mejoras } \\
\text { estadísticamente } \\
\text { significativas } \\
\text { en el número } \\
\text { de respuestas } \\
\text { correctas. Pero no } \\
\text { se hallaron cambios } \\
\text { significativos en } \\
\text { comportamientos } \\
\text { estereotipados. }\end{array}$ \\
\hline $\begin{array}{l}\text { Vander } \\
\text { y Sprong } \\
(2011)\end{array}$ & $\begin{array}{l}\text { Intervención, } \\
\text { cross-over/ } \\
\text { Diagnóstico de } \\
\text { TEA / } \\
3 \text { semanas + } \\
3 \text { semanas. } \\
5 \text { días/ sem. } \\
\text { Antes de clase }\end{array}$ & $\begin{array}{l}9 \text { chicos } \\
\text { ( } 2 \text { chicas)/ } \\
3-6 / \\
\text { USA }\end{array}$ & $\begin{array}{l}2 \text { grupos: } \\
\text { GE ( } n=9) \text { : } \\
\text { 15min correr, } \\
\text { seguido una } \\
\text { tarea en el } \\
\text { aula. } \\
\text { GC }(n=9)\end{array}$ & $\begin{array}{l}\text { Comportamientos } \\
\text { estereotípicos y } \\
\text { comporta-miento } \\
\text { en la tarea }\end{array}$ & $\begin{array}{l}\text { Mejoras en el } \\
\text { número de fallos } \\
\text { y aprendizaje } \\
\text { académico ( } \mathrm{P}< \\
\text { 0,05), pero no en } \\
\text { comportamientos } \\
\text { estereotipados. }\end{array}$ \\
\hline $\begin{array}{l}\text { Liu y } \\
\text { Hamilton } \\
(2013)\end{array}$ & $\begin{array}{l}\text { Intervención/ } \\
\text { Edad, sexo } \\
\text { y trastorno. } \\
\text { Diagnóstico } \\
\text { TEA/ } \\
4 \text { semanas. } 1 \\
\text { día/ sem } \\
\text { Antes de clase }\end{array}$ & $\begin{array}{l}23 \text { niños y } \\
\text { adolescentes } \\
\text { ( } 6 \text { chicas)/ } \\
\text { 5-13/ } \\
\text { USA }\end{array}$ & $\begin{array}{l}1 \text { grupo: } \\
\text { Intervención: } \\
\text { AFMV durante } \\
15 \text { min al día. }\end{array}$ & $\begin{array}{l}\text { Comporta-miento } \\
\text { estereotípico } \\
\text { y durante la Tarea: } \\
\text { observado durante } \\
\text { dos horas y media } \\
\text { cada día } \\
\text { FC }\end{array}$ & $\begin{array}{l}\text { El niño fue } \\
\text { observado durante } \\
\text { dos horas y media } \\
\text { cada día y sus } \\
\text { comportamientos } \\
\text { se clasificaron como } \\
\text { comportamiento } \\
\text { estereotipado o } \\
\text { comprometido } \\
\text { con la tarea. No } \\
\text { hay diferencias } \\
\text { significativas de } \\
\text { comportamiento } \\
\text { relacionadas edad, } \\
\text { sexo y tipo de } \\
\text { trastorno }\end{array}$ \\
\hline $\begin{array}{l}\text { Ketcheson } \\
\text { et al. } \\
\text { (2017) }\end{array}$ & $\begin{array}{l}\text { Intervención/ } \\
\text { Diagnóstico } \\
\text { con ADOS-2 } \\
\text { y DSM-IV/ } \\
8 \text { semanas. } \\
5 \text { días/ sem. } \\
\text { Vacaciones } \\
\text { escolares }\end{array}$ & $\begin{array}{l}20 \text { chicos (5 } \\
\text { chicas)/ } \\
4-6 / \\
\text { USA }\end{array}$ & $\begin{array}{l}2 \text { grupos: } \\
\text { GE ( } n=11) \text { : } \\
\text { Instrucción } \\
\text { de habilidad } \\
\text { motora } \\
\text { durante } 4 \text { h / } \\
\text { día, } \\
\text { GC }(n=9)\end{array}$ & $\begin{array}{l}\text { VABS-2: } \\
\text { comportamiento }\end{array}$ & $\begin{array}{l}\text { Efecto significativo } \\
\text { del tiempo } \\
\text { (en minutos } \\
\text { decrecientes) en } \\
\text { solitario ( } F(4,8,76) \\
=7,94, p<0,01 \text { ) } \\
\text { (Tabla } 5 \text { ). No se } \\
\text { encontró efecto } \\
\text { del tiempo en } \\
\text { comportamiento } \\
\text { (POPE). }\end{array}$ \\
\hline
\end{tabular}

Nota: TEA = Trastorno del Espectro Autista. DSM= Diagnostic and Statistical Manual of Mental Disorders. ADOS= Autism Diagnostic Observation Schedule. GE= Grupo Experimental. GC= Grupo Control. AF= Actividad Física. AFMV= Actividad Física Moderada-Vigorosa. BOSS= Behavioral Observation of Students in Schools. VABS= Vineland Adaptive Behavior Scale. POPE= Playground Observation of Peer Engagement. 


\section{Medida del comportamiento de los niños}

El comportamiento fue medido mediante cuestionarios validados basados en la observación del comportamiento en los niños en la escuela (Nicholson et al., 2011), con Adaptative Behavior Scale y con Playground Observation of Peer Engagement (Ketcheson et al., 2017). En otros casos, se emplearon técnicas observacionales de los comportamientos estereotipados _ balanceo de la manos y brazos, balanceo del cuerpo o movimientos imprecisos descontrolados — (Liu y Hamilton, 2013; Oriel et al., 2011; Vander y Sprong, 2011).

\section{Efecto a medio plazo de la AF en el comportamiento}

Un total de cinco estudios longitudinales con intervención analizaron el efecto a medio plazo de la AF en el comportamiento. El estudio de Liu y Hamilton (2013) analizaron el efecto de la AF realizando una intervención de corta duración a intensidad moderada-vigorosa. Comprobaron que tras cuatro sesiones diferentes de AF de diferente índole pero igual intensidad —-15 min/día a 100-140 latidos/ minuto de intensidad - se producían mejoras en comportamientos estereotipados y en tareas de aprendizaje, no habiendo diferencias atendiendo a edad, sexo, o subtipo de autismo. Un programa de AF basado en la práctica de gran variedad de habilidades motrices - 4 h/día, 5 días/semana, durante 8 semanas_-, mostro mejoras en el comportamiento en niños de entre cuatro y seis años y especialmente en locomoción, habilidades de control de objetos y autocontrol (Ketcheson et al. 2017). Nicholson et al. (2011) hallaron que niños que realizaban un programa de AF justo antes de comenzar la jornada escolar —17 minutos/día, 3 días/semana, durante 2 semanas_ presentaban una actitud más activa hacia el aprendizaje y mejorando el proceso enseñanza-aprendizaje. Sin embrago, Oriel et al. (2011) tras una intervención de carrera aeróbica previa a la jornada escolar — 15 minutos/día, 5 días/semana, durante 3 semanas — no hallaron mejoría en comportamiento y conductas estereotipadas. Finalmente, Vander y Sprong (2011) igualmente revelaron que los niños de tres a seis años que realizaban carrera aeróbica antes de comenzar las clases —-15min/día, 3 días/ semana, durante 3 semanas — no mejoraban el comportamiento en conductas estereotipadas.

\section{DISCUSIÓN Y CONCLUSIONES}

Esta revisión ha analizado los resultados de los trabajos que han estudiado el efecto a medio plazo de un programa de AF en el ámbito escolar, sobre la variable conductual y comportamental en niños menores de 12 años diagnosticados TEA. Los estudios desde enero del 2010 hasta enero 2018 fueron obtenidos de tres bases de datos. Un total de cinco artículos cumplieron los criterios de inclusión, y todos usaron un diseño con intervención. El $60 \%$ de los estudios han mostrado efectos positivos de la AF sobre el comportamiento y el $40 \%$ no hallaron diferencias significativas. El estudio de Liu y Hamilton (2013) mostró que sesiones de ejercicio físico a intensidad moderada-vigorosa, durante 15-30 minutos, tiene un efecto positivo en el comportamiento y conductas estereotipadas/ 
repetitivas. Al igual que otros dos estudios, mostraron efecto positivo de la AF, en cambio, los trabajos de Oriel et al. (2011) y Vander y Sprong (2011) no obtuvieron diferencias significativas positivas.

Observamos que los beneficios de la AF difieren en función del tiempo e intensidad de la intervención, y que sus efectos a medio plazo presentan características diferentes que merecen ser analizadas por separado. La escasez de estudios específicos con niños diagnosticados TEA ha dificultado la posibilidad de hacer comparaciones directas. Trabajar con población de niños autistas en ámbito escolar y deportivo es complejo, debido a la necesidad de formación en atención a la diversidad de los instructores para acometer adecuadamente su labor enseñanza-aprendizaje. Si se incorpora el factor de AF en las clases, la formación y preparación de estos docentes debería de ampliarse, de tal modo que los programas de intervención no interfieran en el currículo escolar. Por tanto, los resultados en ocasiones han sido discutidos en base a los hallazgos obtenidos en intervenciones realizadas fuera del ámbito escolar o en las que también participan adolescentes, así como estudios en los que participa población sin dificultades de aprendizaje (Craig et al., 2016).

El análisis del efecto de una sesión de AF a intensidad moderada-vigorosa, producía mejoras en la cognición, comportamiento y conductas esterotipadas y repetitivas en niños autistas tras cuatro sesiones (Liu y Hamilton, 2013). Resultados coincidentes con los obtenidos por Anderson-Hanley et al. (2011) al someter a 24 adolescentes (10-18 años) diagnosticados con TEA a un estímulo de exergames (videojuegos activos), el grupo experimental mejoró significativamente en comportamiento y conducta [Gilliam Autism Rating Scale, 2nd edition]. En población diagnosticada TDAH, Flohr, Saunders, Evans y Raggi (2004) realizaron un estudio similar, donde 90 niños (7-11 años) realizaron 25 minutos de pedaleo en cicloergómetro a diferentes intensidades, observando efecto positivo al terminar el esfuerzo en el humor, actitud activa, y colaborativa, pero no encontraron diferencias significativas entre los grupos que realizan el ejercicio a intensidad moderada (40-50\%) o vigorosa (65-75\% Frecuencia Cardiaca Máxima). Observamos que otros dos estudios obtuvieron mejoras significativas en el comportamiento de los jóvenes autistas (Ketcheson et al., 2017; Nicholson et al., 2011). Estos resultados son similares a los obtenidos por Brand, Jossen, Holsboer-Trachsler, Pühse y Gerber (2015), que al finalizar el programa de AF de tres semanas de duración — 60 minutos/día, 3 días/semana — observaron mejoras en el comportamiento, eficacia del sueño, humor, así como en aumento de las habilidades motrices. Sin embargo, los otros dos estudios similares incluidos en este trabajo de revisión no observaron diferencias en el comportamiento (Oriel et al., 2011; Vander y Sprong, 2011). No obstante, ninguno de los estudios ha revelado asociación negativa.

Algunos hechos explicarían los efectos a medio plazo del ejercicio físico en el comportamiento y conductas estereotipadas y repetitivas. Al participar en una sesión de AF se produce un incremento del nivel de noradrenalina y dopamina en el encéfalo que provoca mejoras en la actividad cerebral (Berse et al., 2015) mejorando las funciones ejecutivas (Leckie et al., 2014). 
Ante el estímulo generado por una AF a inten-sidad moderada-vigorosa prolongada en el tiempo se produce una respuesta biológica de adaptación de las funciones del cerebro (Raichlen y Polk, 2013). La práctica de ejercicio tiene también efecto relajante, ya que disminuye los niveles de cortisol, bajando niveles de ansiedad y estrés (Hillier et al., 2011), mejorando la calidad del sueño y el humor, y favoreciendo la mejora de comportamiento en niños diagnosticados TEA (Hillier et al., 2011). Destacar la importancia de mejo-rar de la eficacia del sueño, debido a que los niños autistas sufren interrupciones del sueño que afectan negativamente a los síntomas y comportamientos propios del TEA (Cohen, Conduit, Lockley, Rajaratnam y Cornish, 2014).

Por otro lado, no se ha hallado diferencias atendiendo a edad, sexo, o subtipo de autismo (Liu y Hamilton, 2013). La mayoría de los estudios analizaron únicamente el efecto directo de la AF sobre la cognición y comportamiento, obteniendo así probablemente resultados sesgados. Estudios llevados a cabo en escolares sin dificultades de aprendizaje, han comprobado la influencia del índice de masa corporal (Sardinha, Marques, Martins, Palmeira y Minderico, 2014) o porcentaje de grasa corporal (Cadenas-Sánchez et al., 2016).

Pensamos que en trabajos futuros se debería de tener en cuenta la actividad realizada durante todo el día, contabilizando actividades extraescolares, actividad durante clase y el tipo de desplazamiento al colegio, o simplemente si durante los fines de semana y periodos no lectivos practican AF, deporte o videojuegos activos. Debido a que los niños autistas suelen presentan niveles de AF más bajos durante la jornada escolar, y realizan menos actividad diaria que los niños sin dificultades de aprendizaje (Pan et al., 2017), sería interesante conocer si en esta población influyen de forma similar las citadas variables.

La presente revisión engloba un total de cinco artículos de alta calidad, estudios longitudinales, con intervención de en el ámbito escolar. En todos ellos participaron niños menores de 12 años, diagnosticados TEA. La realización de AF a intensidad moderada-vigorosa, ha mostrado en tres casos un efecto positivo en el comportamiento y conductas estereotipadas/repetitivas, dos estudios no obtuvieron efecto positivo en dichas variables. Se emplearon principalmente intervenciones de carácter previo a la jornada escolar. Estos estudios reflejan además que los participantes mejoraron el rendimiento cognitivo y académico, eficacia del sueño, mejorías en el humor y gran desarrollo de habilidades motrices y condición física. Más investigaciones son necesarias para justificar el efecto diferencial a corto, medio y largo plazo en comportamiento y conducta en niños autistas, así como el posible efecto de variar el tipo de ejercicio, intensidad, duración y frecuencia. Los resultados han de ser considerados con cautela, debido a que en su mayor parte han sido obtenidos a partir de muestras pequeñas y con escasa inclusión de posibles variables confusoras.

Los niños autistas precisan los beneficios que el movimiento les aporta para disminuir su ansiedad y estrés, mejora su humor, tener una actitud más activa y colaborativa en clase, así como mejorar el control de comportamientos estereotipados y repetitivos. Atendiendo a los resultados de los estudios revisados en 
este trabajo, se recomienda la práctica diaria de $A F$, en la que se trabaje a una intensidad moderada-vigorosa, incluyendo ejercicios cardiorrespiratorios y práctica de habilidades motrices generales y específicas. Se sugiere realizar antes de cada jornada escolar al menos 15-30 minutos de ejercicio físico variado, pudiendo introducir el componente social y motivacional con juegos en grupo que ayuden a socializarse, incluso mediante el empleo de videojuegos activos o de realidad virtual. A medio plazo serían más evidentes las mejoras en el comportamiento, influyendo además en el rendimiento cognitivo y académico, y especialmente en la condición física y habilidades motrices de todos los niños en general y los niños autistas en particular.

\section{AGRADECIMIENTOS}

Los autores desean agradecer a la Universidad de Jaén la colaboración en el estudio. Este artículo ha sido apoyado en parte por el Programa de Docentes Universitarios, ejecutado por el Ministerio de Educación, Cultura y Deporte del Gobierno de España [FPU-2016-07226].

\section{REFERENCIAS BIBLIOGRÁFICAS}

Altenburg, T. M., Chinapaw, M. J. M., y Singh, A. S. (2015). Effects of one versus two bouts of moderate intensity physical activity on selective attention during a school morning in Dutch primary schoolchildren: A randomized controlled trial. Journal of Science and Medicine in Sport, 19(10), 820-824. doi: 10.1016/j. jsams.2015.12.003

Anderson-Hanley, C., Tureck, K., y Schneiderman, R. L. (2011). Autism and exergaming: effects on repetitive behaviors and cognition. Psychology research and behavior management, 4, 129. doi: 10.2147/PRBM. S24016

American Psychiatric Association (2013). Diagnostic and Statistical Manual of Mental Disorders (DSM-5®). American Psychiatric Pub.

Berse, T., Rolfes, K., Barenberg, J., Dutke, S., Kuhlenbaumer, G., Volker, K., ..., Knecht, S. (2015). Acute physical exercise improves shifting in adolescents at school: evidence for a dopaminergic contribution. Frontiers in Behavioral Neuroscience, 9, 9. doi: 10.3389/fnbeh.2015. 00196

Brand, S., Jossen, S., Holsboer-Trachsler, E., Pühse, U., y Gerber, M. (2015). Impact of aerobic exercise on sleep and motor skills in children with autism spectrum disorders - a pilot study. Neuropsychiatric Disease and Treatment, 11, 1911-1920. doi: 10.2147/ndt.s85650

Cadenas-Sánchez, C., Mora-González, J., Migueles, J. H., Martín-Matillas, M., Gómez-Vida, J., Escolano-Margarit, M. V., ... de Teresa, C. (2016). An exercise-based randomized controlled trial on brain, cognition, physical health and mental health in overweight/obese children (ActiveBrains project): Rationale, design and methods. Contemporary Clinical Trials, 47, 315-324. doi: 10.1016/j.cct.2016.02.007 
Cohen, S., Conduit, R., Lockley, S. W., Rajaratnam, S. M. W., y Cornish, K. M. (2014). The relationship between sleep and behavior in autism spectrum disorder (ASD): a review. Journal of neurodevelopmental disorders, 6(1), 44. doi: 10.1186/1866-1955-6-44

Craig, F., Margari, F., Legrottaglie, A. R., Palumbi, R., de Giambattista, C., y Margari, L. (2016). A review of executive function deficits in autism spectrum disorder and attention-deficit/hyperactivity disorder. Neuropsychiatric Disease and Treatment, 12, 1191-1202. doi: 10.2147/ NDT.S104620

Cacho, C. D., \& Vera, E. C. (2017). Relación entre la danza libre-creativa y autoestima en la etapa de educación primaria. Cuadernos de Psicología del Deporte, 17(1), 73-79.

Fedewa, A. L., y Erwin, H. E. (2011). Stability balls and students with attention and hyperactivity concerns: Implications for on-task and in-seat behavior. American Journal of Occupational Therapy, 65(4), 393-399. doi: 10.5014/ ajot.2011.000554

Flohr, J. A., Saunders, M. J., Evans, S. W., y Raggi, V. (2004). Effects of physical activity on academic performance and behavior in children with ADHD. Medicine and Science in Sports and Exercise, 36(5), S145-S146.

González Hernández, J., y Baños Audije, L. M. (2012). Estudio sobre el cambio de actitudes hacia la discapacidad en clases de actividad física. Cuadernos de Psicología del Deporte, 12(2), 101-108.

Heyvaert, M., Saenen, L., Campbell, J. M., Maes, B., y Onghena, P. (2014). Efficacy of behavioral interventions for reducing problem,behavior in persons with autism: An updated quantitative synthesis of single-subject research. Research in Developmental Disabilities, 35(10), 2463-2476. doi:10.1016/j. ridd.2014.06.017

Hillier, A., Murphy, D., y Ferrara, C. (2011). A Pilot Study: Short-term Reduction in Salivary Cortisol Following Low Level Physical Exercise and Relaxation among Adolescents and Young Adults on the Autism Spectrum. Stress and Health, 27(5), 395-402. doi:10.1002/smi.1391

Ketcheson, L., Hauck, J., y Ulrich, D. (2017). The effects of an early motor skill intervention on motor skills, levels of physical activity, and socialization in young children with autism spectrum disorder: A pilot study. Autism, 21(4), 481-492. doi: $10.1177 / 1362361316650611$

Khan, N. A., y Hillman, C. H. (2014). The relation of childhood physical activity and aerobic fitness to brain function and cognition: a review. Pediatric exercise science, 26(2), 138-146. doi: 10.1123/pes.2013-0125

Lai, M. C., Lombardo, M. V., y Baron-Cohen, S. (2014). Autism. Lancet, 383(9920), 896-910. doi: 10.1016/s0140-6736(13)61539-1

Leckie, R. L., Oberlin, L. E., Voss, M. W., Prakash, R. S., Szabo-Reed, A., Chaddock-Heyman, L., ... Erickson, K. I. (2014). BDNF mediates improvements in executive function following a 1-year exercise intervention. Frontiers in $\mathrm{Hu}$ - 
man Neuroscience, 8, 12. doi:10.3389/ fnhum.2014.00985

Liu, T., y Hamilton, M. (2013). The effects of physical activity on the stereotypic behaviors of children with autism spectrum disorder. Journal of Sport \& Exercise Psychology, 35, S68-S68. doi: 10.17795/intjsh-28674

Nicholson, H., Kehle, T. J., Bray, M. A., y Heest, J. V. (2011). The effects of antecedent physical activity on the academic engagement of children with autism spectrum disorder. Psychology in the Schools, 48(2), 198-213. doi: 10.1002/ pits. 20537

Oriel, K. N., George, C. L., Peckus, R., y Semon, A. (2011). The effects of aerobic exercise on academic engagement in young children with autism spectrum disorder. Pediatric Physical Therapy, 23(2), 187-193. doi: 10.1097/ PEP.0b013e318218f149

Ortega, F. B., Artero, E. G., Ruiz, J. R., España-Romero, V., Jiménez-Pavón, D., Vicente-Rodríguez, G., ... Ottevaere, C. (2011). Physical fitness levels among European adolescents: the HELENA study. British journal of sports medicine, 45(1), 20-29. doi: 10.1136/bjsm.2009.062679

Pan, C. Y., Chu, C. H., Tsai, C. L., Sung, M. C., Huang, C. Y., y Ma, W. Y. (2017).

The impacts of physical activity intervention on physical and cogntive outcomes in children with autism spectrum disorder. Autism: The International Journal Of Research And Practice, 21(2), 190-202. doi: 10.1177/ 1362361316633562

Piepmeier, A T, Shih, C. H., Whedon, M., Williams, L. M., Davis, M. E., Henning, D. A., ... Etnier, J. L. (2015). The effect of acute exercise on cognitive performance in children with and without ADHD. Journal of Sport and Health Science, 4(1), 97-104. doi: 10.1016/j.jshs.2014.11.004

Raichlen, D. A., y Polk, J. D. (2013). Linking brains and brawn: exercise and the evolution of human neurobiology. Proceedings of the Royal Society of London B: Biological Sciences, 280(1750), 9. doi: 10.1098/rspb.2012.2250

Sánchez-Meca, J., y Botella, J. (2010). Revisiones sistemáticas y meta-análisis: Herramientas para la práctica profesional. Papeles del Psicólogo, 31(1), 7-17.

Sardinha, L. B., Marques, A., Martins, S., Palmeira, A., y Minderico, C. (2014). Fitness, fatness, and academic performance in seventh-grade elementary school students. BMC pediatrics, 14(1), 1. doi: 10.1186/1471- 2431-14-176

Schmidt, M., Benzing, V., y Kamer, M. (2016). Classroom-based physi-cal activity breaks and children's attention: Cognitive engagement works! Frontiers in psychology, 7, 1-13. doi: 10.3389/fpsyg.2016.01474

Sowa, M., y Meulenbroek, R. (2012). Effects of physical exercise on Autism Disorders: A meta-analysis. Research in Autism Spectrum Disorders, 6, 4657. doi: 10.1016/j.rasd.2011.09.001

Suarez-Manzano, S., Ruiz-Ariza, A., De La Torre-Cruz, M., \& Martínez-López, E. J. (2018). Acute and chronic effect of physical activity on cogni-tion and behaviour in young people with ADHD: A systematic review of intervention studies. Research in developmental disabilities, 77, 12-23. doi: 10.1016/ j.ridd.2018.03.015 
Vander Net, J., y Sprong, M. (2011). Clinical bottom line. Commentary on "The effects of aerobic exercise on academic engagement in young children with autism spectrum disorder." Pediatric Physical Therapy, 23(2), 187-193. doi: 10.1097/PEP.0b013e3182193630 